\title{
Behaviour-Aided Environment Detection for Context Adaptive Navigation
}

\author{
Han Gao, University College London (UCL)
}

\section{BIOGRAPHY}

Han Gao is a PhD student at University College London in the Engineering Faculty's Space Geodesy and Navigation Laboratory (SGNL). He received a Bachelor's degree in Aerospace Engineering from Shanghai Jiao Tong University (SJTU) in 2014. He is interested in multi-sensor contextual navigation, machine learning and smartphone based positioning techniques.

\begin{abstract}
Navigation and positioning systems dependent on both the operating environment and the behaviour of the host vehicle or user. The environment determines the type and quality of radio signals available for positioning and the behaviour can contribute additional information to the navigation solution. In order to operate across different contexts, a context-adaptive navigation solution is required to detect the operating contexts and adopt different positioning techniques accordingly. This paper focuses on detecting both environmental and behavioural contexts as a whole with smartphone sensors, serving for a context-adaptive navigation system. Behavioural contexts cover both human activities and different vehicle motions. The performance of behaviour recognition in this paper is improved by feature selection and a connectivity dependent filter. Environmental contexts are detected from GNSS measurements. They are classified into indoor, intermediate and outdoor categories using a probabilistic support vector machine, followed by a hidden Markov model used for time-domain filtering. As there will never be completely reliable context detection, the paper also shows how behaviours can be associated within environment detection to improve the reliability of context determination algorithms. Finally, the proposed context-determination algorithms are tested in a series of multi-context scenarios.
\end{abstract}

\section{INTRODUCTION}

Navigation and positioning are inherently dependent on the context, which comprises both the operating environment and the behaviour of the host vehicle or user [1]. Nowadays, many navigation systems are required to operate in different contexts. For our smartphones, their contexts may change from indoor to outdoor environments and can be stationary, on a pedestrian, or on any type of vehicle. To deliver better positioning services, the consideration of context is essential for a navigation system. Although many techniques have been developed or improved for greater navigation accuracy and reliability, their operating scenarios are subject to limited contexts. Examples include multiple-constellation global navigation satellite systems (GNSS) [2][3], indoor Wi-Fi fingerprinting [4][5], 3D-mapping aided (3DMA) GNSS [6][7][8], and pedestrian dead reckoning (PDR) using step detection [9]. To make full use of these techniques in practical applications, it is necessary to integrate them and then implement the most suitable ones based on context.

In order to meet the demand of providing more accurate and reliable positioning services across a wide range of contexts, a multi-sensor integrated navigation solution is therefore required, which is able to detect the operating context and configure the positioning system accordingly. This is also referred to as context-adaptive navigation [1][10].

Environmental and behavioural context respectively reveal where the system is and what the user is doing under particular circumstances. On one hand, the environment determines the type and quality of available radio signals for navigation. For example, most radio signals do not propagate at all in an underwater environment. GNSS reception is good in open-sky environments but poor indoors and in deep urban areas, therefore the corresponding processing techniques also vary. Wi-Fi and Bluetooth signals are not available in rural areas, in the air or at sea. Visual positioning performs best in the environments with lots of features. On the other hand, the behaviour can contribute additional information to the navigation solution. A stationary pedestrian or land vehicle indicates a fixed location and will not need to update its velocity and position. Trains normally remain on the track, effectively constraining the possible positioning solutions. The requirements of navigation (e.g. accuracy, availability and update rate) can also vary with behaviours.

Context determination is the basis of context-adaptive navigation. Previous work on context detection focused on improving individual subsystems. For instance, cognitive GNSS has been investigated to adjust the processing strategies and parameters of GNSS receivers [11][12][13][14]. Behaviour recognition and sensor location detection has been implemented to improve PDR [15][16][17]. There has also been substantial research into activity classification for indoor positioning applications [18][19][20]. Environment detection focused on differentiating between indoor and outdoor environment. Using the "IODetector" proposed by Zhou [21], indoor/outdoor environment is determined by using a combination of cellular signals, light sensors, magnetometers 
and proximity sensors. With the same sensor combinations, semi-supervised learning [22] and sequential models [23] have been further considered to improve the classification accuracy. Besides, a detailed outdoor environment framework for urban and open-sky area detection has been proposed in [24].

Although behavioural and environmental context can be detected separately, they are not completely independent in reality [1]. For example, a car typically travels on the road, not in the air or at the bottom of the sea. Previous research focused on improving behaviour recognition with environmental context. For example, human behaviour was analysed in [25] by integrating location and emotion context. In [26], the authors presented a location-aware activity recognition approach utilising a Bayesian Network, suggesting the accuracy of activity recognition can be improved with environmental features. A Location-MotionContext (LoMoCo) model was proposed in [27], which uses Bayes reasoning to infer human behaviour by estimating the probability of motion patterns occurring at the locations. A similar framework was presented in [28] under hidden Markov models, where the pedestrian location and motion states are used in a reciprocal manner to improve their estimation of one another. An upgraded version of the model was further described in [29] by introducing multiple aspects of context for inferring human activities.

Preliminary environmental and behavioural context detection research has been presented in [30], laying the foundation of context detection. Building upon the previous work, this paper contributes to context determination from two aspects. First, a context determination framework is proposed, within which both behavioural and environmental context can be detected, filtered and associated for a reliable context determination. Second, distinct from other research using environment information for better behaviour classification performances, this paper shows how environment detection can be improved with the aid of behaviour recognition, since the environment can provide more indication of the signals and positioning techniques applicable for contextadaptive navigation. The rest of the paper is structured as follows. Section 2 describes the framework and processes of context determination for adaptive navigation system. Section 3 considers behaviour recognition, including behavioural feature selection and time-domain filter. Section 4 proposes the investigation of environment detection, including classification and smoothing. Section 5 investigates how to improve the reliability of environment determination by making use of behaviour information. Section 6 then presents and compares the performance of different context detection algorithms under various scenarios. The conclusions are summarized in Section 7.

\section{CONTEXT DETERMINATION FRAMEWORK}

Figure 1 shows the overall framework of the context detection algorithm. Firstly, both time and frequency domain features are extracted from the sensor measurements. Behaviour features are extracted from accelerometers, gyroscopes, magnetometers and the barometer on the smartphone while environments will be detected based on GNSS signals. The classification models are constructed from the training datasets and able to distinguish the corresponding context categories for the test input features. The outputs of the classification models shall be estimated as probabilities, so that the following context determination processes and navigation system can adopt different strategies according to the certainty of the results. Then, both behaviour and environment classification results from the models are filtered by the proposed behavioural connectivity method and hidden Markov model respectively, aiming to minimise incorrect context determination by considering the time-domain relationship between successive epochs. To further enhance the context determination reliability, behaviour and environment association will be considered within the algorithm. Shown as dash lines in Figure 1, context associations include applying different features and classification models based on the subject of the behaviours, and adjusting the parameters of the hidden Markov model using the probabilities of the behaviours. Finally, the results of context determination can be used to select suitable sensors, positioning algorithms or subsystems for better navigation performance.

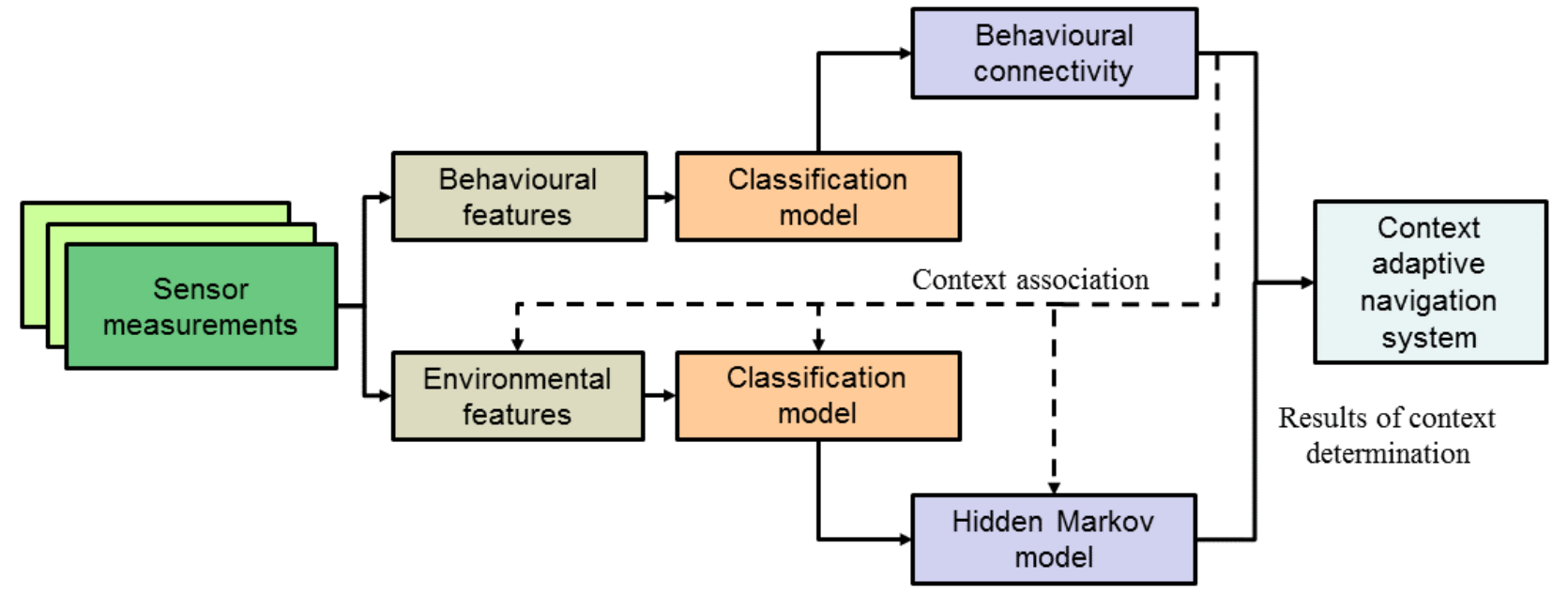

Figure 1. Diagram of context detection algorithm 


\section{BEHAVIOURAL CONTEXT RECOGNITION}

A two-step process is implemented for behavioural context recognition: pattern recognition and connectivity. Pattern recognition algorithms are used to assess the probabilities of the behaviour belonging to each category, then the connectivity mechanism further improves the accuracy of recognition by estimating the relationship of behaviour categories between successive epochs.

An implementation of pattern recognition algorithms has been described in detail in [30]. In the paper, a hierarchy framework was first developed to distinguish both pedestrian activities and vehicle motions, which are shown in Figure 2. Different supervised machine learning algorithms were applied for classification using the features extracted from accelerometers, gyroscopes, magnetometers and the barometer. By comparing with others, decision tree (DT) shows better performance for human-vehicle classifier, while relevance vector machine (RVM) was selected for the other two classifiers.

This section will further extend the previous work in [30]. In Section 3.1, feature selection algorithms will be applied to find the best combination of features that can perform better classification results. To enhance the recognition reliability, the connectivity algorithm will be introduced in Section 3.2.

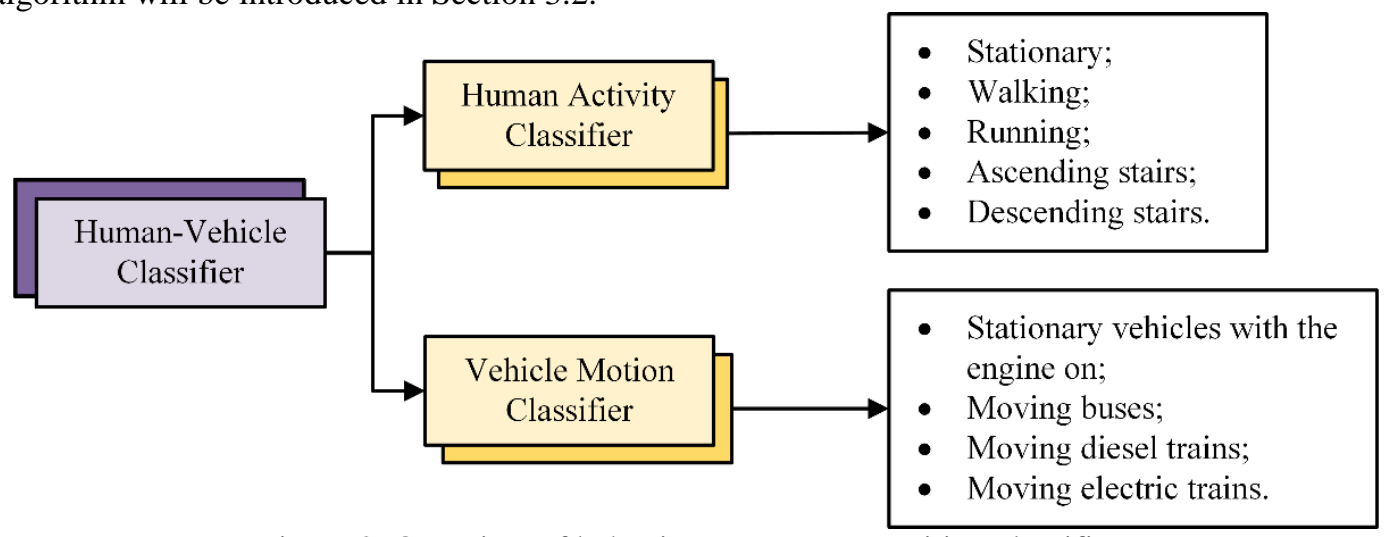

Figure 2. Overview of behaviour pattern recognition classifiers

\subsection{Features Selection and Classification}

In [30], both time and frequency domain features were extracted from sensor measurements. They are summarized in Table 1. Using all of the proposed features, the corresponding classification accuracies of the human-vehicle, human activity and vehicle motion classifiers were $98.9 \%, 97.6 \%$ and $91.0 \%$, respectively.

However, some of the extracted features may be redundant, introducing noise and irrelevant information. This can cause a deterioration of the classification performance. In order to identify the most relevant features for distinguishing different activities, feature selection techniques are therefore used and described. To explore the best combination of features, the Sequential Forward Floating Selection (SFFS) algorithm [31] is investigated in this paper. The SFFS algorithm aims to minimise the misclassified samples over all feasible feature subsets to obtain better classification performance.

The SFFS consists of two parts: a new feature for the current feature subset is added if better classification performance is achieved; a conditional exclusion is then applied to the new feature subset, from which the least significant feature is determined. If the least significant feature is the last one added, the algorithm goes back to select a new feature. Otherwise the least significant feature is excluded and moved back to the available feature subsets and conditional exclusion is continued. This cycle is repeated until there is no further improvement of classification performance. The advantage of this method is that the discarded features can be selected again in the inclusion and exclusion procedure.

(a) human-vehicle classifier

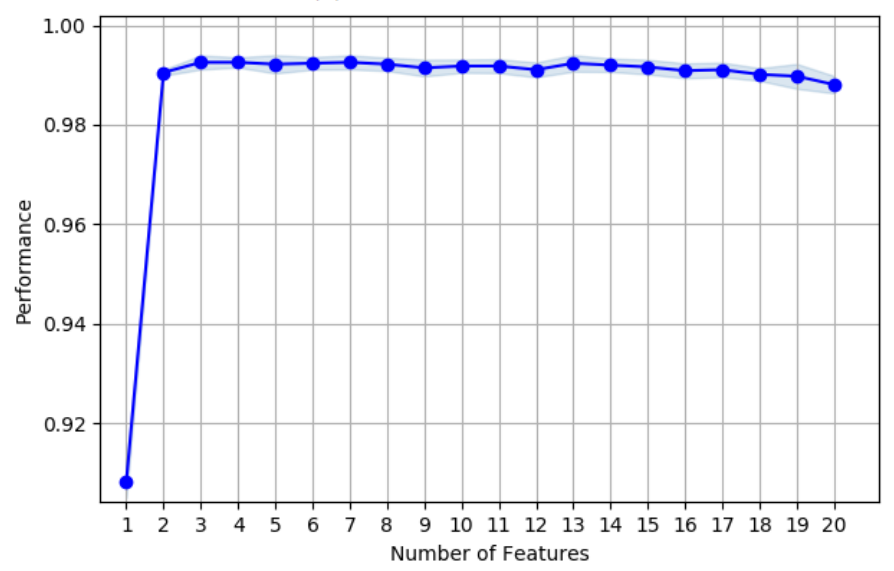

(b) human activity classifier

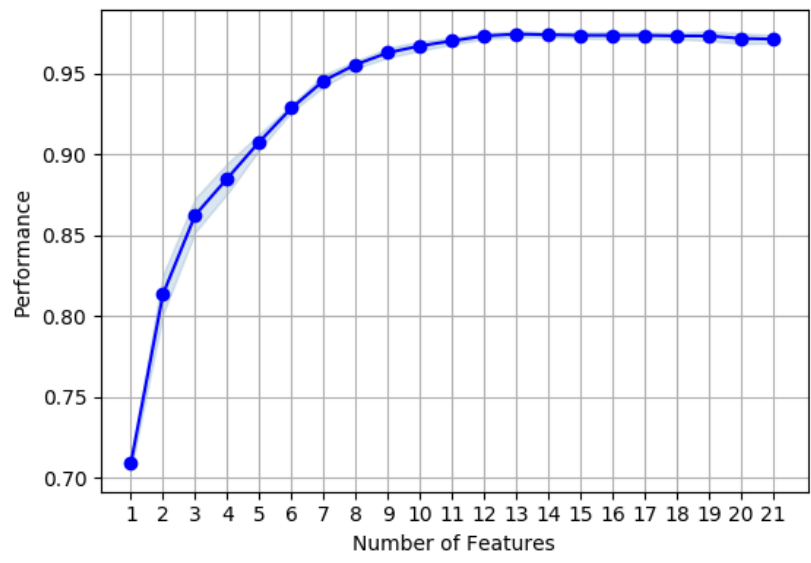


(c) vehicle motion classifier

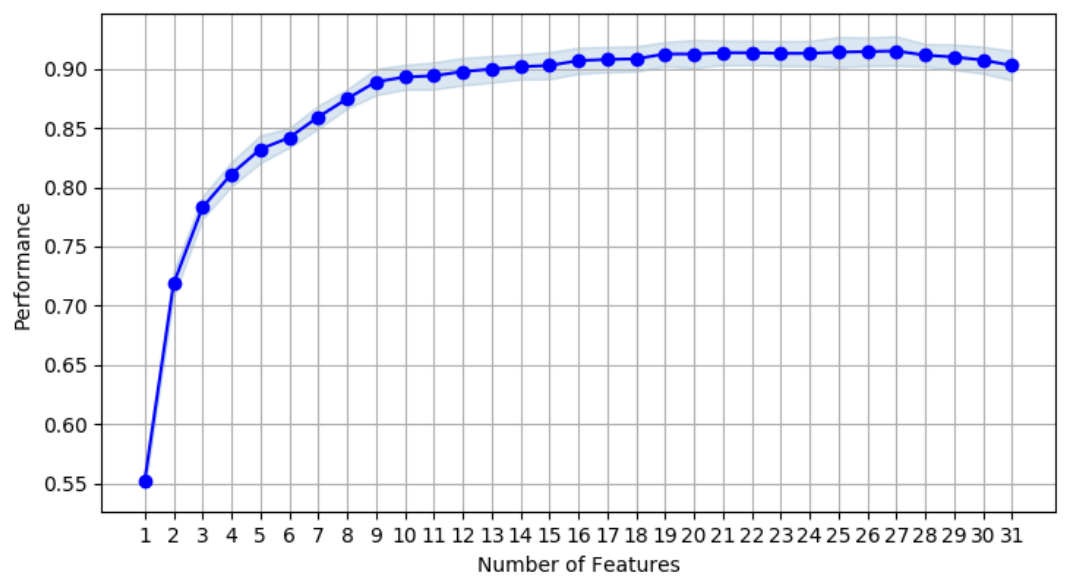

Figure 3. Feature selection performances for three classifiers

Table 1. A summary of behavioural features (features selected by SFFS are marked in blue)

\begin{tabular}{|c|c|c|c|c|c|}
\hline & & Expression & $\begin{array}{l}\text { Human-Vehicle } \\
\text { Classifier }\end{array}$ & $\begin{array}{l}\text { Human Activity } \\
\text { Classifier }\end{array}$ & $\begin{array}{l}\text { Vehicle Motion } \\
\text { Classifier }\end{array}$ \\
\hline \multirow{17}{*}{ 离 } & F1 & range $_{\text {acc }}$ & $\sqrt{ }$ & $\sqrt{ }$ & $\sqrt{ }$ \\
\hline & F2 & range $_{\text {gyro }}$ & $\sqrt{ }$ & $\sqrt{ }$ & $\sqrt{ }$ \\
\hline & $\mathbf{F 3}$ & range $_{\text {magn }}$ & $\sqrt{ }$ & $\sqrt{ }$ & $\sqrt{ }$ \\
\hline & F4 & range $_{\text {baro }}$ & $\sqrt{ }$ & $\sqrt{ }$ & $\sqrt{ }$ \\
\hline & F5 & $\overline{\sigma_{a c c}}$ & $\sqrt{ }$ & $\sqrt{ }$ & $\sqrt{ }$ \\
\hline & F6 & $\sigma_{g y r o}$ & $\sqrt{ }$ & $\sqrt{ }$ & $\sqrt{ }$ \\
\hline & F7 & $\sigma_{m a g n}$ & $\sqrt{ }$ & $\sqrt{ }$ & $\sqrt{ }$ \\
\hline & F8 & $\sigma_{\text {baro }}$ & $\sqrt{ }$ & $\sqrt{ }$ & $\sqrt{ }$ \\
\hline & F9 & skewness ${ }_{a c c}$ & $\sqrt{ }$ & $\sqrt{ }$ & $\sqrt{ }$ \\
\hline & F10 & skewness gyro & $\sqrt{ }$ & $\sqrt{ }$ & $\sqrt{ }$ \\
\hline & F11 & skewness ${ }_{\text {magn }}$ & $\sqrt{ }$ & $\sqrt{ }$ & $\sqrt{ }$ \\
\hline & F12 & skewness $_{\text {baro }}$ & $\sqrt{ }$ & $\sqrt{ }$ & $\sqrt{ }$ \\
\hline & F13 & kurtosis $_{\text {acc }}$ & $\sqrt{ }$ & $\sqrt{ }$ & $\sqrt{ }$ \\
\hline & F14 & kurtosis gyro $_{\text {gyr }}$ & $\sqrt{ }$ & $\sqrt{ }$ & $\sqrt{ }$ \\
\hline & F15 & kurtosis $s_{\text {magn }}$ & $\sqrt{ }$ & $\sqrt{ }$ & $\sqrt{ }$ \\
\hline & F16 & kurtosis $_{\text {baro }}$ & $\sqrt{ }$ & $\sqrt{ }$ & $\sqrt{ }$ \\
\hline & F17 & $Z Z_{\text {Zacc }}$ & & $\sqrt{ }$ & \\
\hline \multirow{7}{*}{ 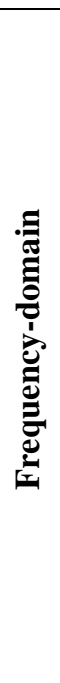 } & F18 & Peak magnitude acc & $\sqrt{ }$ & $\sqrt{ }$ & \\
\hline & F19 & Peak magnitude gyro & $\sqrt{ }$ & $\sqrt{ }$ & \\
\hline & F20 & Frequency index of F18 & $\sqrt{ }$ & $\sqrt{ }$ & \\
\hline & F21 & Frequency index of F19 & $\sqrt{ }$ & $\sqrt{ }$ & \\
\hline & $\begin{array}{l}\text { F22- } \\
\text { F26 }\end{array}$ & $\begin{array}{l}\text { Peak magnitudes of acc in sub- } \\
\text { bands: } 0-10 \mathrm{~Hz}(\mathrm{~F} 22), 10-20 \mathrm{~Hz} \\
(F 23), 20-30 \mathrm{~Hz}(\mathrm{~F} 24), \quad 30- \\
40 \mathrm{~Hz}(\mathrm{~F} 25), 40-50 \mathrm{~Hz}(\mathrm{~F} 26)\end{array}$ & & & $\begin{array}{c}\sqrt{ } \\
\text { (F22-F25 are } \\
\text { selected by SFFS) }\end{array}$ \\
\hline & $\begin{array}{l}\text { F27- } \\
\text { F31 }\end{array}$ & $\begin{array}{l}\text { Peak magnitudes of gyro in sub- } \\
\text { bands: } 0-10 \mathrm{~Hz}(\mathrm{~F} 27), 10-20 \mathrm{~Hz} \\
(F 28), 20-30 \mathrm{~Hz}(\mathrm{~F} 29), \quad 30- \\
40 \mathrm{~Hz}(\mathrm{~F} 30), 40-50 \mathrm{~Hz}(\mathrm{~F} 31)\end{array}$ & & & $\begin{array}{c}\sqrt{ } \\
(\mathrm{F} 27, \mathrm{~F} 28, \mathrm{~F} 30, \\
\text { F31 are selected } \\
\text { by SFFS) }\end{array}$ \\
\hline & $\begin{array}{l}\text { F32- } \\
\text { F36 }\end{array}$ & PSD of acc in sub-bands & & & $\begin{array}{c}\sqrt{ } \\
(\mathrm{F} 32, \mathrm{~F} 33, \mathrm{~F} 35, \\
\text { F36 are selected } \\
\text { by SFFS) }\end{array}$ \\
\hline
\end{tabular}


The SFFS algorithm was implemented for behavioural recognition, by using the same dataset and the same corresponding machine learning algorithms for each classifier described in [30]. Figure 3 shows the average classification accuracy of the three classifiers as a function of the number of features selected by SFFS. The shadow area in the figure indicates the standard deviation using 5-fold cross validation. The results show that the classification performance of each classifier can be slightly improved from 98.9\% (20 features, human-vehicle classifier), 97.6\% (21 features, human activity classifier) and $91.0 \%$ ( 31 features, vehicle motion classifier) to $99.3 \%$ (4 features), $97.9 \%$ (13 features) and 91.5\% (27 features) respectively. The dimensions of features are reduced at the same time.

\subsection{Connectivity}

Behaviour connectivity describes the time-domain relationship between the current and previous behaviour categories. The transitions between some behaviours are more likely than others [1]. For example, stationary vehicles and pedestrian behaviours can be connected directly, whereas moving behaviour of different vehicles is not because a vehicle must normally stop to enable a person to get in or out.

Figure 4 shows the behaviour connectivity algorithm, comprising the following five steps at each epoch. Full details are presented in [30].

1. A smoothing method is first applied to compute the provisional estimates of behaviour probabilities at current epoch by combining the normalized outputs from the pattern recognition algorithms with the estimates at last epoch;

2. The relationship between current and previous behaviours can then be expressed as a transfer matrix, calculated from the estimated probabilities, $\hat{\mathbf{x}}_{k}^{-}$and $\hat{\mathbf{x}}_{k-1}$;

3. In a connectivity matrix, the likelihoods of connections between behaviours can be preset based on whether a direct transition could happen between them in practice. Thus the transfer matrix can be re-estimated using the connectivity matrix;

4. Then the likelihoods of the behavioural estimates at current epoch $\hat{\mathbf{x}}_{k}^{+}$are updated by multiplying the previous behavioural probabilities $\hat{\mathbf{x}}_{k-1}$ by the new transfer matrix;

5. Finally, the probability of each behaviour at current epoch $\hat{\mathbf{x}}_{k}$ is obtained by re-scaling the likelihoods $\hat{\mathbf{x}}_{k}^{+}$.

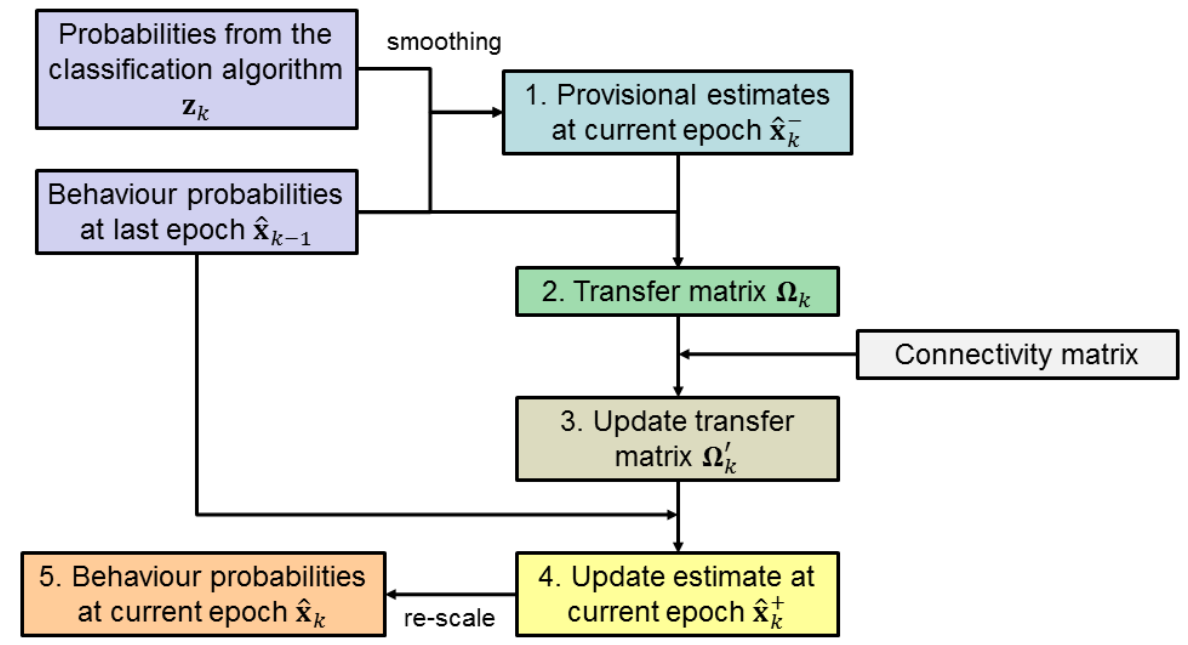

Figure 4. Block diagram of behaviour connectivity

\section{ENVIRONMENTAL CONTEXT DETECTION}

Different kinds of radio signals are inherently environment-dependent and may be used for environment classification. The section begins by proposing the categorisation of environments and explaining why GNSS signals have been selected for detecting the environments. The extraction of suitable features based on the availability and strength of GNSS signals is then described. Next, the environments are classified by a probabilistic support vector machine (SVM) in contrast to the heuristic approach described in [30]. Then, the SVM outputs will be smoothed by a hidden Markov model (HMM) to improve the accuracy of context determination.

\subsection{Categorisation}

A good environment categorization for navigation is expected to provide an indication of the positioning techniques applicable for determining position in that environment. For most land navigation applications, locating whether the user is indoor or outdoor is a prerequisite task because indoor and outdoor positioning depend on inherently distinct techniques. In an outdoor environment, GNSS or enhanced GNSS techniques perform well while Wi-Fi positioning or Bluetooth positioning are better options when staying inside a building. In reality, the boundaries between indoor and outdoor environments can be ambiguous, rendering some scenarios hard to classify as either indoor or outdoor [30]. Thus, the intermediate environment, where a client is adjacent to a building or in a partially enclosed environment, is included as one of the categories. Note that the 
occurrence of intermediate scenarios is quite rare for vehicles, so this category is ignored when the subject is identified as a vehicle. Typical examples of each environment category for both pedestrian and vehicle are shown in Figure 5.
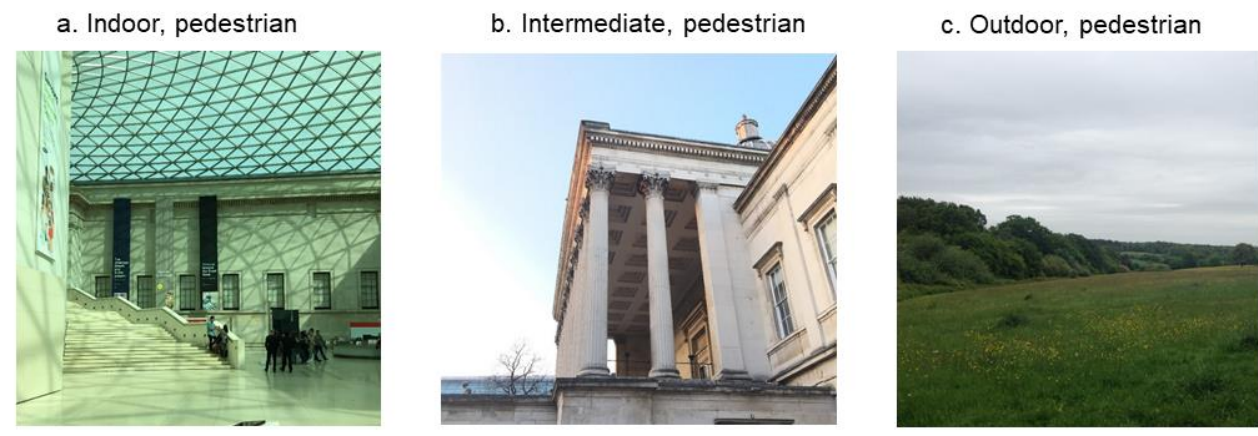

d. Indoor, vehicle

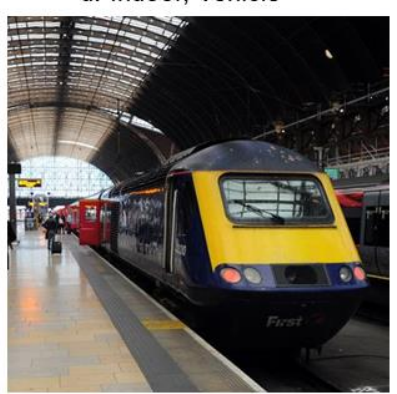

e. Outdoor, vehicle

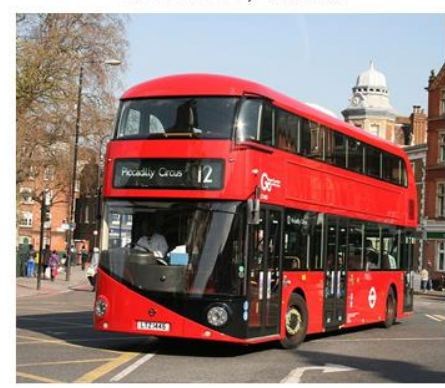

Figure 5. Examples of different environment categories

GNSS signals are used for environment detection in this study for two reasons. First, among smartphone sensors, the availability and quality of satellite signals tend to be less affected by factors other than the environment type. For example, Wi$\mathrm{Fi}$, Bluetooth and cellular signals strongly rely on the allocation density of the broadcasting station. Their signal strengths also depend on the distance away from the stations, whereas GNSS signal strengths are roughly constant across the Earth's surface. Second, the globally distributed properties of GPS and GLONASS ensure that we can infer environments from the availability and strength of GNSS signals anywhere on Earth. The full development of Galileo and Beidou System in the future will further enhance the detection performance. The main drawback of GNSS is its high-power consumption when constantly updated. As the research advances, other sensors could be added into the context determination framework to improve upon the environment detection using the GNSS module.

\subsection{Feature Extraction}

In an indoor environment, most GNSS signals are attenuated by the structure of the building or received by non-line-of-sight (NLOS) paths, rendering them weaker (or unavailable) indoors compared with intermediate and outdoor environments. Thus features comprising the total number of satellites received and the total measured carrier-power-to-noise-density ratio $\left(\mathrm{C} / \mathrm{N}_{0}\right)$ summed across all satellites received at each epoch are extracted.
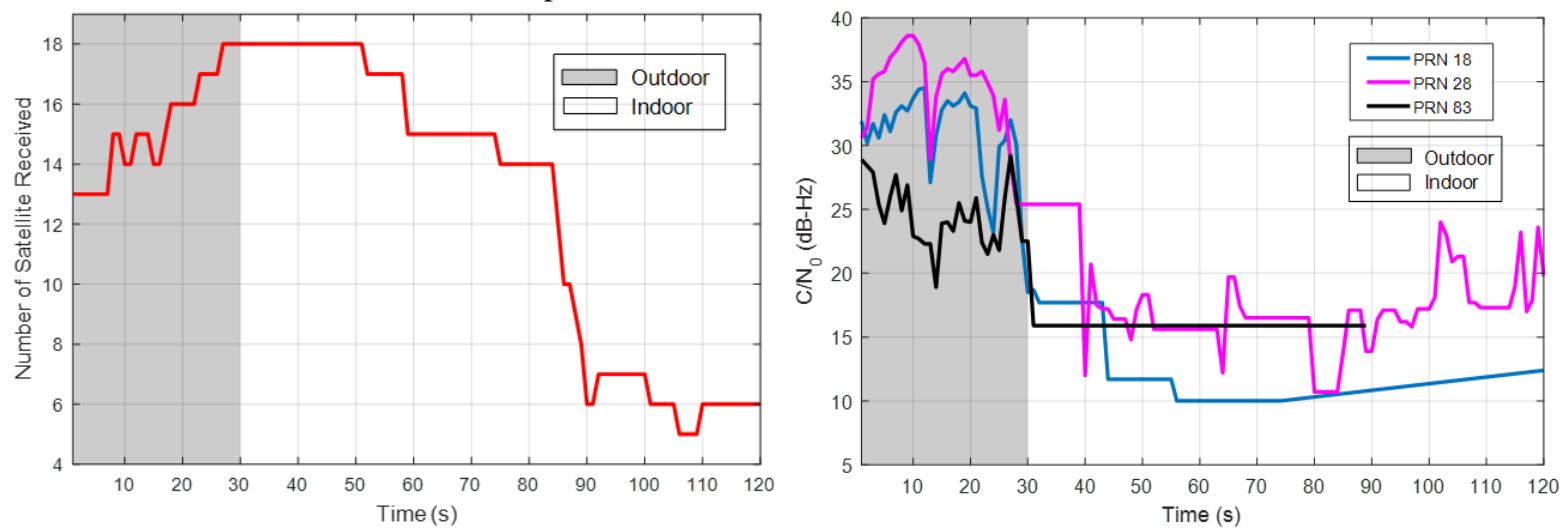

Figure 6. Number of satellites received (left) and selected $\mathrm{C} / \mathrm{N}_{0}$ values (right) during an indoor-outdoor transition

To show the effectiveness of the proposed features, a set of GNSS measurements was collected on the smartphone. The person holding the smartphone kept walking from an outdoor into an indoor environment. The transition happened at about the 30th second. Figure 6 demonstrates the differences in availability and strength of GNSS signals, respectively, in the indoor and 
outdoor environments. The number of satellites received decreased gradually after moving indoors, as more satellite signals were blocked by the building. Concerning the $\mathrm{C} / \mathrm{N}_{0}$ outputs from the selected three satellites, a drop of about $5 \mathrm{~dB}-\mathrm{Hz}$ was observed when the person was nearing the building, following by a sharp decrease when they entered. It was also noted that most of the satellite signals indoors were weaker than $20 \mathrm{~dB}-\mathrm{Hz}$ and more satellites lost track after $80 \mathrm{~s}$ as the person moved deeper indoors.

However, based on the findings in [30], it is difficult to distinguish "shallow indoor" and "deep urban" from each other with only these two features, so more metrics are required for a reliable indoor/outdoor classification. The extraction of additional features is discussed in detail in Section 5.1.

\subsection{Probabilistic Support Vector Machine}

The SVM is a supervised classification algorithm derived from statistical learning theory and kernel based methods [32][33]. Given the training samples with corresponding target labels $\left(y_{i} \in\{-1,+1\}\right)$, the SVM can construct the classification hyperplane in the high-dimensional feature space that maximises the margin between two classes and minimises the error.

Suppose $f\left(\boldsymbol{x}_{k}\right)$ is the output of the SVM that measures the distance between the test sample $\boldsymbol{x}_{k}$ and the hyperplane. Platt [34] proposed an approach to obtain the classification probability by fitting the SVM output with a sigmoid function:

$$
P\left(y_{k}=+1 \mid \mathbf{x}_{k}\right)=\frac{1}{1+\exp \left(A f\left(\mathbf{x}_{k}\right)+B\right)} .
$$

The parameters $A$ and $B$ of Equation (1) are found using maximum likelihood estimation from the training data and their corresponding target values. It is worth to note that the training set can be but does not have to be the same set as used for training the SVM [35].

Fundamentally, the SVM is a binary classifier. This is sufficient to classify two vehicle environment categories. To extend the method for pedestrian environment classification situations with three environment categories, three binary classifiers can be trained. Each one is created to separate every two environment categories. Using Platt's method for each SVM we get, these pairwise probabilities are combined into posterior probabilities following [36]

$$
P\left(S_{i} \mid \mathbf{x}_{k}\right)=1 /\left[\sum_{j=1, j \neq i}^{L} \frac{P\left(S_{i} \text { or } S_{j} \mid \mathbf{x}_{k}\right)}{P\left(S_{i} \mid \mathbf{x}_{k}\right)}-(L-2)\right]
$$

where $S_{i}$ denotes the environment context in this research and there are $L=3$ environmental contexts for pedestrian cases.

\subsection{Hidden Markov Model}

A hidden Markov model is a time-sequential pattern recognition algorithm, which assumes a Markov process [37] with the states. It is capable of modelling the movements of a smartphone from one environment to another according to observations in this study. Within an HMM, the probabilities that the system is in each of the states are estimated, so that the navigation system knows the certainty of the decision. In general, an HMM comprises five elements as follows:

1) The state space $S$ consists of three hidden states: indoor, intermediate and outdoor, which are denoted as $S_{1}, S_{2}$ and $S_{3}$ respectively. At each epoch $k$, the hidden states satisfy the condition

$$
\sum_{i=1}^{L} P\left(\mathrm{Z}_{k}=S_{i}\right)=1
$$

where $Z_{k}$ is the environmental context at that epoch and $L$ refers to the number of environment categories.

2) The set of observations $\boldsymbol{x}_{k}$ at each epoch $k$, comprises the extracted features for environment detection.

3) The matrix of state transition probabilities $\mathbf{A}=\left\{A_{i j}\right\}$. Each element of the state transition probabilities matrix, $A_{i j}$, defines the probability that a state $S_{i}$ at the immediately prior epoch transits to another state $S_{j}$ at the current epoch.

4) The matrix of emission probabilities $\mathbf{B}=\left\{B_{i}(k)\right\}$ that defines the conditional distributions $\mathrm{P}\left(\boldsymbol{x}_{k} \mid S_{i}\right)$ of the observations from a specific state.

5) An initial state probability distribution $\pi=\left\{\pi_{i}\right\}$ that defines the probability of state $S_{i}$ being at the first epoch.

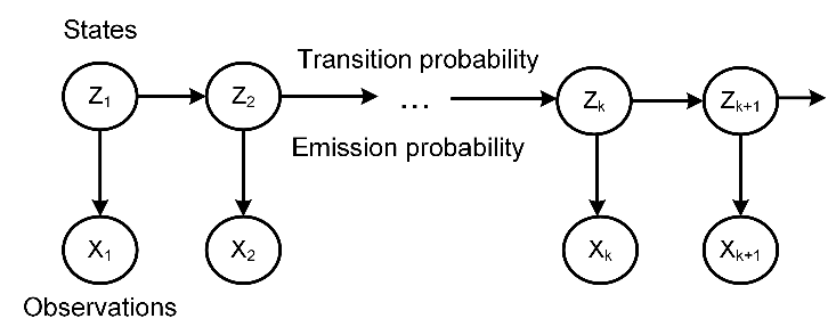

Figure 7. Structure of a first-order HMM

The first-order HMM is implemented in environment detection, which suggests the current environmental context is only affected by the immediate previous context. Figure 7 is an illustration of a first-order hidden Markov model. Given the sequence 
of the observations, the probabilities of each context at each epoch can be inferred using the Viterbi algorithm [33][37]. The probabilities of the HMM proposed for pedestrian situation with three environment contexts are determined as follows.

Initial probability. As there is no prior information about the initial state, we have to make a judgement based on the available initial observations. Clearly, the indoor and outdoor contexts occur much more frequently than the intermediate context. However, if there is insufficient information to correctly determine the context, it is better to select the intermediate context than to incorrectly select the indoor or outdoor context. The initial probabilities were therefore set as follows:

$$
\begin{aligned}
& P\left(Z_{1}=S_{1}\right)=P\left(Z_{1}=S_{3}\right)=0.4 \\
& P\left(Z_{1}=S_{2}\right)=0.2
\end{aligned}
$$

Transition probability. Since the sample interval here is $1 \mathrm{~s}$, when a user was previously indoors, the current state is highly likely to be indoor and might be intermediate, but is not likely to be outdoor. This is because the user rarely moves directly from indoors to a fully outdoor GNSS reception environment. However, when the user is at the intermediate state, he/she can move directly to either of the other states. Based on these assumptions and with reference to the parameters applied in IODetector [21], the values of the transition probability were as listed in Table 2. Note that the values are selected in order to obtain an experimental balance between responsiveness to change and vulnerability to noise.

Table 2. Transition probabilities of $\operatorname{HMM}\left(\mathbf{A}_{0}\right)$

\begin{tabular}{cccc}
\hline $\begin{array}{c}\text { previous } \\
\text { current }\end{array}$ & Indoor & Intermediate & Outdoor \\
\hline Indoor & $2 / 3$ & $1 / 3$ & 0 \\
Intermediate & $1 / 3$ & $1 / 3$ & $1 / 3$ \\
Outdoor & 0 & $1 / 3$ & $2 / 3$ \\
\hline
\end{tabular}

Emission probability. The emission probability describes the measurement likelihood of making an observation in different states. Given the test samples $\boldsymbol{x}_{k}$, the emission probability here can be transformed from the probabilistic outputs by the SVM in Equation (2) by using Bayes' rule:

$$
P\left(\mathbf{x}_{k} \mid S_{i}\right) \propto \frac{P\left(S_{i} \mid \mathbf{x}_{k}\right)}{P\left(S_{i}\right)}
$$

where $\mathrm{P}\left(S_{i}\right)$ is the prior probability of the class. Their values are set the same as the initial probabilities in Equation (4).

\section{CONTEXT ASSOCIATION}

Although behavioural and environmental context are detected separately, they are not completely independent [1]. Certain environments are associated with certain behaviours. For example, all road vehicles are associated with driving, but only offroad vehicles are associated with off-road driving. A bus typically travels more slowly and stops more in cities than on the highway. The location of a stationary pedestrian will not change until they move. This information can be used to estimate the likelihood of the detected behaviour and environment combinations and reduce the chances of the context determination algorithms selecting an incorrect context.

In this section, the process of environment detection will be assisted with behaviour information in two different ways. They are explored in Section 5.1 and 5.2 respectively.

\subsection{Transition Probability of HMM}

The transition matrix $\mathbf{A}_{0}$ given by Table 2 is proposed for general cases without considering the behaviours of the users. In reality, a stationary user will stay in the same environment, making it impossible to transit from one to another. Therefore, the stationary probability from behaviour recognition results is used to modify the transition probability, as shown in Equation (6).

$$
\mathbf{A}=p_{\text {stat }} \mathbf{I}+\left(1-p_{\text {stat }}\right) \mathbf{A}_{0}
$$

where $\mathbf{I}$ is the identity matrix and $p_{\text {stat }}$ denotes the detected probability of being stationary for both a pedestrian and vehicle.

If the user is stationary $\left(p_{\text {stat }}=1\right)$, the transition matrix will be equal to the identity matrix, indicating an unchanged environment; if the user is detected to be moving $\left(p_{\text {stat }}=0\right)$, the transition proposed for general cases will be used in HMM.

\subsection{Pedestrian/Vehicle Association}

The strengths of GNSS signals are different inside a vehicle than for a pedestrian. Figure 8 shows the normalized distributions of GNSS $\mathrm{C} / \mathrm{N}_{0}$ inside a vehicle and on a pedestrian under indoor scenarios. They were both collected statically for about 10 minutes at London Paddington train station. Two sites were about $1 \mathrm{~m}$ away from each other, so they can be treated as similar indoor scenarios. It can be seen that the average signal strength of the vehicle data is about $10 \mathrm{~dB}-\mathrm{Hz}$ weaker than the corresponding pedestrian one, as a result of attenuation by the vehicle's body. Therefore, different environment classification models should be implemented for vehicles and pedestrians. 

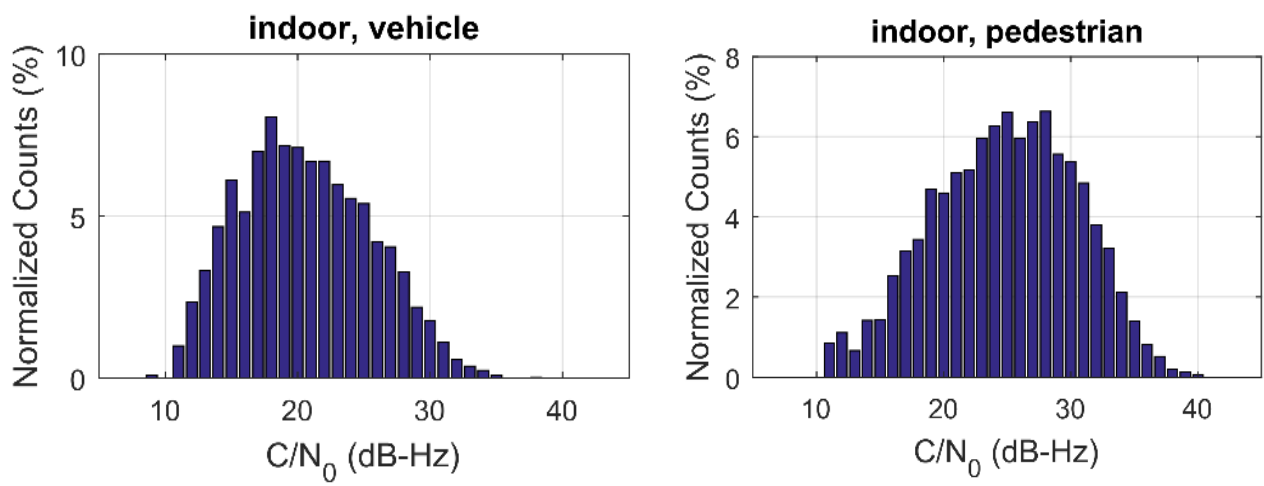

Figure 8. Comparison of vehicle and pedestrian indoor data (both data were collected at London Paddington train station, the vehicle data was collected inside a Heathrow Express train and the pedestrian one was collected by the train about $1 \mathrm{~m}$ away from the inside point)

As proposed in [30], the feature that total $\mathrm{C} / \mathrm{N}_{0}$ values summed across the satellite signals above $25 \mathrm{~dB}-\mathrm{Hz}$, was proven to be effective in indoor/outdoor classification for a pedestrian. Therefore, in addition to the two features proposed in Section 4.2, it is adopted as a third feature for pedestrian based environment classification, denoted as sumCNR $\mathrm{R}_{25}$.

We also consider the application of a similar metric for environment classification inside vehicles, so features with various $\mathrm{C} / \mathrm{N}_{0}$ thresholds were assessed. A 5-fold cross-validation strategy of the vehicle dataset that will be described in Section 6.1 was applied to find the optimum threshold with the best classification accuracy. The three input features to the SVM are the total $\mathrm{C} / \mathrm{N}_{0}$ values summed across the satellite signals above different thresholds, along with the total number of received satellites and total signal strength of the GNSS signals. The classification performance obtained with different thresholds is shown in Table 3 . The feature, total $\mathrm{C} / \mathrm{N}_{0}$ values summed across the satellite signals above $30 \mathrm{~dB}-\mathrm{Hz}$, shows better performances than others and is thus selected for vehicle based environment classification. It is denoted as $\operatorname{sumCNR} 30$.

Table 3. Classification performance with respect to different thresholds

\begin{tabular}{c|c}
\hline Threshold & Accuracy \\
\hline $20 \mathrm{~dB}-\mathrm{Hz}$ & $80.6 \%$ \\
\hline $25 \mathrm{~dB}-\mathrm{Hz}$ & $80.3 \%$ \\
\hline $30 \mathrm{~dB}-\mathrm{Hz}$ & $82.4 \%$ \\
\hline $35 \mathrm{~dB}-\mathrm{Hz}$ & $80.6 \%$ \\
\hline
\end{tabular}

A summary of the features for both pedestrian and vehicle based environment classification is presented in Table 4. The HMM parameters in Section 4.4 are proposed for pedestrian situations. Since the environmental categorisation of vehicle is different from the pedestrian, the corresponding initial and transition probabilities are adjusted for vehicle situations in Equation (7) and Table 5 respectively, while the emission probabilities can still be obtained from Equation (5).

$$
P\left(Z_{1}=S_{1}\right)=P\left(Z_{1}=S_{3}\right)=0.5
$$

Table 4. Features for environment classification

\begin{tabular}{c|c}
\hline Pedestrian & Vehicle \\
\hline \multicolumn{2}{c}{ Total number of satellites received } \\
\hline \multicolumn{2}{c}{ Total measured $\mathrm{C} / \mathrm{N}_{0}$ values } \\
\hline sumCNR $_{25}$ & sumCNR $_{30}$ \\
\hline
\end{tabular}

Table 5. Transition probabilities for vehicle-based $\operatorname{HMM}\left(\mathbf{A}_{0}\right)$

\begin{tabular}{ccc}
\hline previous & Indoor $\left(\mathbf{S}_{\mathbf{1}}\right)$ & Outdoor $\left(\mathbf{S}_{\mathbf{3}}\right)$ \\
\hline Indoor & $2 / 3$ & $1 / 3$ \\
Outdoor & $1 / 3$ & $2 / 3$ \\
\hline
\end{tabular}




\section{EXPERIMENT RESULTS AND DISCUSSION}

In this section, different application scenarios were used to test the performance of the proposed context detection system. Firstly, the collection of the training dataset is described in Section 6.1. In Section 6.2 and Section 6.3, the performances of the pedestrian and vehicle experiments under different kinds of scenarios are examined and compared with other methods.

\subsection{Environment Training Dataset}

The environment dataset was collected using a Google Pixel smartphone running Android data logging applications. Both GPS and GLONASS data was logged at $1 \mathrm{~Hz}$. GNSS measurements, comprising time tags, PRN (pseudo-random number) of the satellites, the $\mathrm{C} / \mathrm{N}_{0}$ measurements, satellite azimuths and elevations can all be logged in files for processing.

To construct the classification models, the training data was collect on a pedestrian or inside a vehicle. Table 6 summaries the dataset that covers different kinds of scenarios in indoor, intermediate and outdoor environments. Note that all the pedestrian environmental data was collect statically.

Table 6. Description of environment training dataset

\begin{tabular}{|l|l|l|l|l|}
\hline Location & Subject & Type of environment & Duration & Note \\
\hline Hyde Park & Pedestrian & Open-sky, outdoor & 30 mins & Collected in Feb. 2017 \\
\hline $\begin{array}{l}\text { Central London, near Fenchurch } \\
\text { street station }\end{array}$ & Pedestrian & Deep urban, outdoor & 50 mins & Collected in Nov. 2016 \\
\hline Different UCL buildings & Pedestrian & Indoor & 90 mins in total & Collected in January 2017 \\
\hline 3 places on UCL Portico & Pedestrian & Intermediate & 30 mins *3 times & $\begin{array}{l}\text { Shown in Figure 5 (b), } \\
\text { Collected in April 2017 }\end{array}$ \\
\hline $\begin{array}{l}\text { Bus 188 route (from Russell } \\
\text { square to Waterloo station) }\end{array}$ & Bus & Outdoor & 20 mins & Collected in June 2017 \\
\hline $\begin{array}{l}\text { Bus 14 route (from Chenies } \\
\text { street to Green Park) }\end{array}$ & Bus & Outdoor & 20 mins & Collected in June 2017 \\
\hline $\begin{array}{l}\text { Srain route from London to } \\
\text { London Paddington train station }\end{array}$ & Train & Outdoor & 10 mins & Collected in June 2017 \\
\hline London Victoria train station & Train & Indoor & 30 mins & Collected in July 2017 \\
\hline
\end{tabular}

\subsection{Kinematic Pedestrian Experiments}

To test the environment detection ability under different GNSS reception conditions, the proposed environment detection methods were examined under four different scenarios. Each scenario was conducted for 20 minutes. They are shown in Figure 9. Among two outdoor scenarios, Scenario One is an open-sky park while Scenario Two is a typical traditional European area with narrow streets and buildings packed close together. It is important to note that all the data collections in this Section were conducted in January 2018. Thus the time between training dataset and the test data was at least longer than half a year, allowing the satellite positions to change significantly. Therefore, all the collected test data are independent of the training dataset. During the data collection, the experimenter was allowed to hold the smartphone and behave as normal within the experimental area, such as walking, running, waiting for traffic, and standing to take photos. At the same time, besides GNSS measurements, the sensor measurements from accelerometers, gyroscopes, magnetometers and the barometer are also recorded for behaviour recognition.

Scenario One (outdoor)

Scenario Two (outdoor) Scenario Three (intermediate)

Scenario Four (indoor)

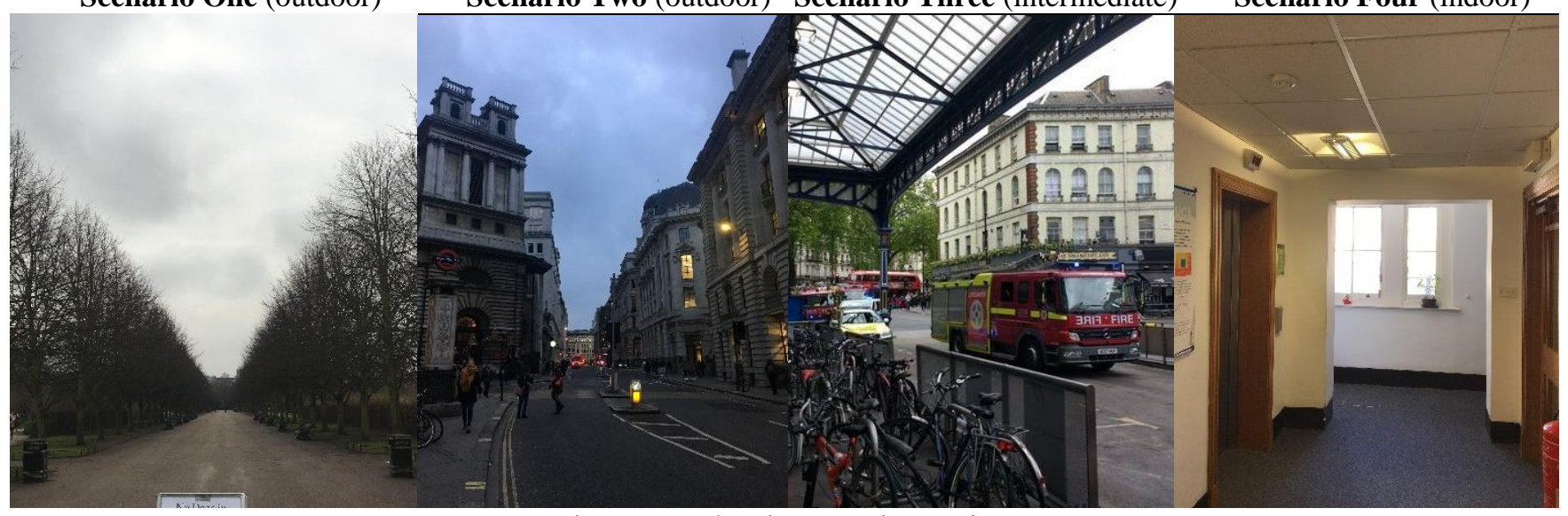

Figure 9. Pedestrian experiment sites 
A GMM-HMM environment detection approach was proposed in [24], whose emission probabilities were modeled by a mixture of Gaussian distributions based on the fitting database. To access the performance, the classification results of GMMHMM, SVM alone, SVM-HMM with and without association (whether adopting the strategy in Section 5.1) are presented and compared in Table 7.

Comparing SVM alone results with the ones smoothed by HMM, it can be seen that a substantial improvement in detecting indoor and outdoor environments is achieved. This shows that the consideration of the time-sequential relationships between environments can improve context detection. Comparing SVM-HMM classification results with and without association shows that the adjustment of transition probabilities with stationary probability does not always improve the environment detection, depending on the situation. When the estimation of the previous epoch is correct, the adjustment is helpful; otherwise, it is not. For the indoor and outdoor classification tasks, the proposed SVM-HMM with association method all perform better than GMMHMM method. Some contexts are more difficult to be distinguished than others. The classification performances of Scenario Three are poor for all approaches, showing the intermediate context is far more difficult to distinguish than indoor and outdoor context.

Table 7. Classification results of pedestrian experiments (1200 samples in total for each scenario)

\begin{tabular}{|c|c|c|c|c|}
\hline & indoor & intermediate & outdoor & accuracy $(\%)$ \\
\hline \multicolumn{5}{|c|}{ Scenario One (Regent's Park, collected on 22/01/2018) } \\
\hline GMM-HMM & 0 & 6 & 1194 & 99.50 \\
\hline SVM & 6 & 0 & 1194 & 99.50 \\
\hline SVM-HMM without association & 4 & 2 & 1194 & 99.50 \\
\hline SVM-HMM with association & 0 & 0 & 1200 & 100 \\
\hline \multicolumn{5}{|c|}{ Scenario Two (Central London near Bank and Monument stations, collected on 12/01/2018) } \\
\hline GMM-HMM & 29 & 177 & 994 & 82.83 \\
\hline SVM & 0 & 153 & 1047 & 87.25 \\
\hline SVM-HMM without association & 0 & 39 & 1161 & 96.75 \\
\hline SVM-HMM with association & 0 & 42 & 1158 & 96.50 \\
\hline \multicolumn{5}{|c|}{ Scenario Three (under the awning of Victoria station, collected on 13/01/2018) } \\
\hline GMM-HMM & 5 & 315 & 880 & 26.25 \\
\hline SVM & 15 & 191 & 994 & 15.92 \\
\hline SVM-HMM without association & 0 & 181 & 1019 & 15.08 \\
\hline SVM-HMM with association & 0 & 239 & 961 & 19.92 \\
\hline \multicolumn{5}{|c|}{ Scenario Four (inside UCL Chadwick building, collected on 13/01/2018) } \\
\hline GMM-HMM & 1194 & 6 & 0 & 99.50 \\
\hline SVM & 979 & 221 & 0 & 81.58 \\
\hline SVM-HMM without association & 1180 & 20 & 0 & 98.33 \\
\hline SVM-HMM with association & 1200 & 0 & 0 & 100.00 \\
\hline
\end{tabular}

\subsection{Vehicle Experiment}

To assess and compare the performances of different approaches on a vehicle, a practical test was conducted on a bus. The bus travelled along South Colonnade Street in the Canary Wharf district of London and stopped at the bus station under the bridge for about 20 seconds, as shown in Figure 10. This route was designed to incorporate both indoor and outdoor environments, as well as moving and stationary vehicle motions.

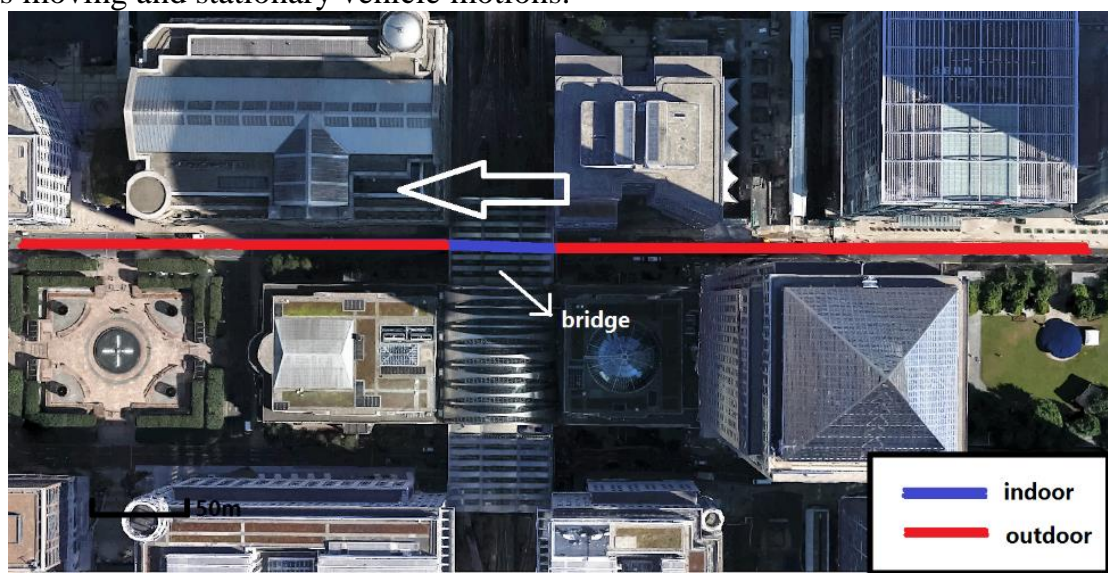

Figure 10. An aerial view of the bus route (satellite image from Google Earth) 
The context detection results are shown in Figure 11. From Figure 11(a), the behavioural detection outputs, most of the samples were correctly detected with behavioural connectivity, showing that connectivity can improve the performance of behaviour recognition. The selection of $4 \mathrm{~s}$ window length for feature selection resulted in about $3 \mathrm{~s}$ in the behaviour recognition, as a balance between accuracy and latency.

From the performance of environment detection, it can be observed that the behaviour-aided SVM determination and SVMHMM methods are able to recognise most indoor and outdoor contexts on the bus, while the GMM-HMM approach failed to detect most outdoor samples especially when the bus travelled into the outdoor environment from indoors. This proves that using association to adopt different (vehicle/pedestrian) models in environment detection according to the recognised behaviour can improve the performance of environment detection in a vehicle. Meanwhile, it is also observed that the combination of HMM and SVM performs slightly better than the SVM method alone. The optimization of HMM transition probabilities by considering the status of the behaviours may further improve the environment detection accuracy.

(a) Behaviour detection results

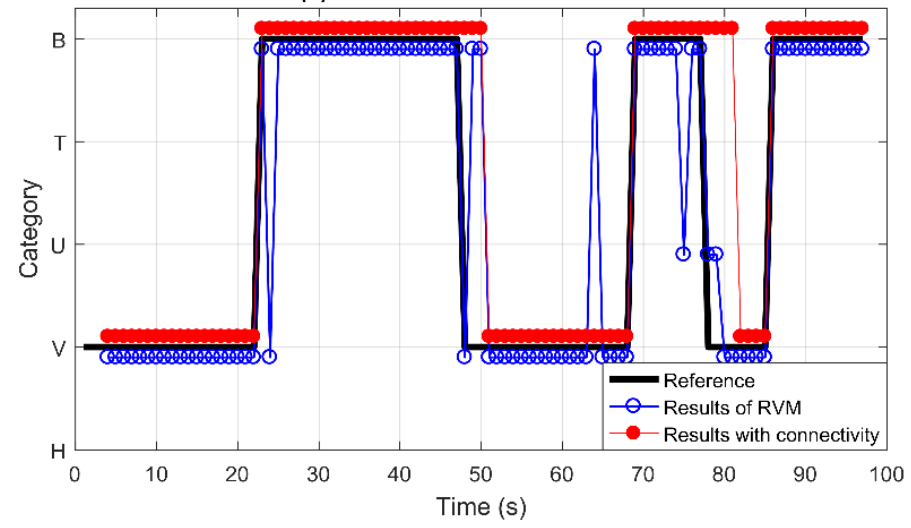

(b) Environment detection results
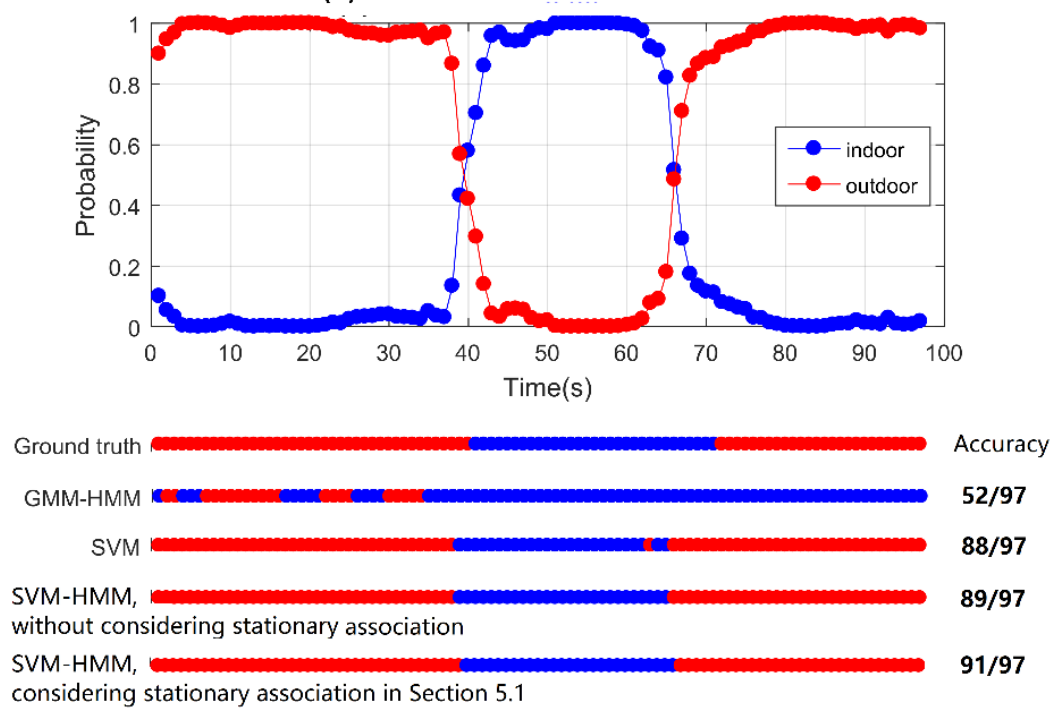

Figure 11. Performance of vehicle context detection

(Note: $\mathrm{B}=$ moving buses, $\mathrm{T}=$ moving diesel trains, $\mathrm{U}=$ moving electric trains, $\mathrm{V}=$ stationary vehicles with the engine on, $\mathrm{H}=$ human activities.)

\section{CONCLUSIONS}

This paper explores environmental context detection with the aid of behavioural context using smartphone sensors. The purpose of context determination is for context-adaptive navigation, that is able to detect the operating contexts and reconfigure the positioning algorithms and subsystems.

The improvement of existing behaviour recognition framework is considered from feature selection and connectivity. Feature selection algorithms were applied to find the best combination of behavioural features. Results showed that feature selection can slightly improve the performance of each sub-classifier within behavioural recognition framework to $99.3 \%$ (4 features), $97.9 \%$ (13 features) and $91.5 \%$ (27 features), with the corresponding dimension of features decreased. The concepts and processes of 
behaviour connectivity have also been described. By exploiting time dependent relationship, connectivity was used to further improve the reliability of behaviour recognition.

Environmental context detection has focused on indoor and outdoor classification. A detection scheme is developed based on GNSS signals and estimating probability of each context. Based on the detected behavioural subject (whether it is on a pedestrian or inside a vehicle), different features of the satellite signals are extracted and trained to construct the SVM classification models. Then a hidden Markov model is used to smooth the results by assuming a Markov process with the environments. As environments and behaviours are not completely independent, context association is considered by using results of behaviour recognition to adopt suitable parameters and classification models within the environment detection process. Practical experiments under different scenarios showed that the proposed method is able to distinguish most indoor and outdoor contexts with over $95 \%$ accuracy for pedestrian and over $90 \%$ accuracy for vehicle.

\section{ACKNOWLEDGMENTS}

The author would like to acknowledge his supervisor Dr. Paul D Groves, for his insights and supportive efforts throughout the $\mathrm{PhD}$ project. This work is funded by the UCL Engineering Faculty Scholarship Scheme and the Chinese Scholarship Council (CSC).

\section{REFERENCES}

[1] Groves, P.D., et al., "Context Detection, Categorization and Connectivity for Advanced Adaptive Integrated Navigation," ION GNSS 2013, Nashville, TN, September 2013, pp. 1039-1056.

[2] Groves, P.D., Principles of GNSS, Inertial, and Multisensor Integrated Navigation Systems, Second Edition, Boston London: Artech House, 2013.

[3] Betz, J.W., Engineering Satellite-Based Navigation and Timing: Global Navigation Satellite Systems, Signals, and Receivers, John Wiley \& Sons, 2015.

[4] Ching, W., Teh, R.J., Li, B., and Rizos, C., “Uniwide WiFi Based Positioning System,” IEEE International Symposium on Technology and Society (ISTAS), June 2010, pp. 180-189.

[5] Bell, S., Jung, W.R., and Krishnakumar, V., "WiFi-Based Enhanced Positioning Systems: Accuracy Through Mapping, Calibration, and Classification," Proceedings of the 2nd ACM SIGSPATIAL International Workshop on Indoor Spatial Awareness, ACM, November 2010, pp. 3-9.

[6] Groves, P.D., "Shadow Matching: A New GNSS Positioning Technique for Urban Canyons," The Journal of Navigation, 64(3), 2011, 417-430.

[7] Adjrad, M., and Groves, P.D., "Enhancing Least Squares GNSS Positioning with 3D Mapping without Accurate Prior Knowledge," NAVIGATION, 64(1), 2017, 75-91.

[8] Groves, P.D., and Adjrad, M., "Likelihood-based GNSS Positioning using LOS/NLOS Predictions from 3D Mapping and Pseudoranges," GPS Solutions, 21(4), 2017, 1805-1816.

[9] Pratama, A.R., and Hidayat, R., "Smartphone-Based Pedestrian Dead Reckoning as an Indoor Positioning System," System Engineering and Technology (ICSET), 2012 International Conference on. IEEE, 2012.

[10] Groves, P.D., et al., "The Four Key Challenges of Advanced Multisensor Navigation and Positioning," IEEE/ION PLANS 2014, Monterey, California, May 2014, pp. 773-792.

[11] Lin, T., O’Driscoll, C., and Lachapelle, G., "Development of a Context-Aware Vector-Based High-Sensitivity GNSS Software Receiver," ION ITM 2011, San Diego, CA, January 2011, pp. 1043-1055.

[12] Shivaramaiah, N.C., and Dempster, A.G., "Cognitive GNSS Receiver Design: Concepts and Challenges," ION GNSS 2011, Portland, OR, September 2011, pp. 2782-2789.

[13] Shafiee, M., O'Keefe, K., and Lachapelle, G., "Context-Aware Adaptive Extended Kalman Filtering using Wi-Fi Signals for GPS Navigation," ION GNSS 2011, Portland, OR, September 2011, pp. 1305-1318.

[14] Lin, T., O'Driscoll, C., and Lachapelle, G., "Channel Context Detection and Signal Quality Monitoring for Vector-Based Tracking Loops," ION GNSS 2010, Portland, OR, September 2010, pp. 1875-1888.

[15] Park, C.G., et al., “Adaptive Step Length Estimation with Awareness of Sensor Equipped Location for PNS,” ION GNSS 2007, Fort Worth, TX, September 2007, pp. 1845-1850.

[16] Shin, S.H., et al., "Pedestrian Dead Reckoning System with Phone Location Awareness Algorithm," IEEE/ION PLANS 2010, Indian Wells, California, July 2010, pp. 97-101.

[17] Martinelli, A., et al., "Probabilistic Context-aware Step Length Estimation for Pedestrian Dead Reckoning," IEEE Sensors Journal, 18(4), 2018, 1600-1611. 
[18] Pei, L., et al. "Using LS-SVM Based Motion Recognition for Smartphone Indoor Wireless Positioning," Sensors, 12(5), 2012, 6155-6175.

[19] Elhoushi, M., et al. "Motion Mode Recognition for Indoor Pedestrian Navigation Using Portable Devices." IEEE Transactions on Instrumentation and Measurement, 65(1), 2016, 208-221.

[20] Radu, V., and Marina, M.K., "HiMLoc: Indoor Smartphone Localization via Activity Aware Pedestrian Dead Reckoning with Selective Crowdsourced WiFi Fingerprinting," IPIN 2013, Montbeliard-Belfort, France, October 2013.

[21] Zhou, P., et al., "IODetector: A Generic Service for Indoor Outdoor Detection," Proceedings of the 10th ACM Conference on Embedded Network Sensor Systems, ACM, November 2012, pp. 113-126.

[22] Radu, V., et al., "A Semi-Supervised Learning Approach for Robust Indoor-Outdoor Detection with Smartphones," Proceedings of the 10th ACM Conference on Embedded Network Sensor Systems, ACM, November 2014, pp. $280-294$.

[23] Li, S., et al., "A Lightweight and Aggregated System for Indoor/Outdoor Detection using Smart Device,” Future Generation Computer Systems, 2017.

[24] Gao, H., and Groves, P.D., "Environmental Context Detection for Adaptive Navigation using GNSS Measurements from a Smartphone," NAVIGATION, 2018. (accepted for publication)

[25] Banos, O., et al. "Human Behavior Analysis by Means of Multimodal Context Mining." Sensors, 16(8), $2016,1264$.

[26] Lu, C.H., and Fu, L.C., "Robust Location-Aware Activity Recognition Using Wireless Sensor Network in an Attentive Home," IEEE Transactions on Automation Science and Engineering, 6(4), 2009, 598-609.

[27] Pei, L., et al. "Human Behavior Cognition using Smartphone Sensors,” Sensors, 13(2), 2013, 1402-1424.

[28] Liu, J., et al. "Reciprocal Estimation of Pedestrian Location and Motion State toward a Smartphone Geo-Context Computing Solution," Micromachines, 6(6), 2015, 699-717.

[29] Chen, R., et al. "Inferring Human Activity in Mobile Devices by Computing Multiple Contexts," Sensors, 15(9), 2015, 21219-21238.

[30] Gao, H., and Groves, P.D., "Context Determination for Adaptive Navigation using Multiple Sensors on a Smartphone," ION GNSS 2016, Portland, Oregon, September 2016, pp. 742-756.

[31] Pudil, P., Novovičová, J., and Kittler, J., "Floating Search Methods in Feature Selection," Pattern Recognition Letters, 15(11), 1994, 1119-1125.

[32] Bishop, C.M., Pattern Recognition and Machine Learning, Company New York, 2006.

[33] Vapnik, V., The Nature of Statistical Learning Theory, Springer, 1995.

[34] Platt, J., "Probabilistic Outputs for Support Vector Machines and Comparisons to Regularized Likelihood Methods," Advances in Large Margin Classifiers, 10(3), 1999, 61-74.

[35] Valstar, M.F., and Pantic, M., "Combined Support Vector Machines and Hidden Markov Models for Modeling Facial Action Temporal Dynamics,” Human-Computer Interaction, 2007, 118-127.

[36] Price, D., et al., "Pairwise Neural Network Classifiers with Probabilistic Outputs," Neural Information Processing Systems, 7, 1995, 1109-1116.

[37] Shoaib, M., et al., "Fusion of Smartphone Motion Sensors for Physical Activity Recognition,” Sensors, 14(6), 2014, 1014610176. 